\title{
Virtual Microphones for Multichannel Audio Resynthesis
}

\author{
Athanasios Mouchtaris \\ Electrical Engineering Systems Department, Integrated Media Systems Center (IMSC), University of Southern California, \\ 3740 McClintock Avenue, Los Angeles, CA 90089-2564, USA \\ Email:mouchtar@sipi.usc.edu

\section{Shrikanth S. Narayanan} \\ Electrical Engineering Systems Department, Integrated Media Systems Center (IMSC), University of Southern California, \\ 3740 McClintock Avenue, Los Angeles, CA 90089-2564, USA \\ Email:shri@sipi.usc.edu

\section{Chris Kyriakakis} \\ Electrical Engineering Systems Department, Integrated Media Systems Center (IMSC), University of Southern California, \\ 3740 McClintock Avenue, Los Angeles, CA 90089-2564, USA \\ Email: ckyriak@imsc.usc.edu
}

Received 30 May 2002 and in revised form 17 February 2003

\begin{abstract}
Multichannel audio offers significant advantages for music reproduction, including the ability to provide better localization and envelopment, as well as reduced imaging distortion. On the other hand, multichannel audio is a demanding media type in terms of transmission requirements. Often, bandwidth limitations prohibit transmission of multiple audio channels. In such cases, an alternative is to transmit only one or two reference channels and recreate the rest of the channels at the receiving end. Here, we propose a system capable of synthesizing the required signals from a smaller set of signals recorded in a particular venue. These synthesized "virtual" microphone signals can be used to produce multichannel recordings that accurately capture the acoustics of that venue. Applications of the proposed system include transmission of multichannel audio over the current Internet infrastructure and, as an extension of the methods proposed here, remastering existing monophonic and stereophonic recordings for multichannel rendering.
\end{abstract}

Keywords and phrases: multichannel audio, Gaussian mixture model, distortion measures, virtual microphones, audio resynthesis, multiresolution analysis.

\section{INTRODUCTION}

Multichannel audio can enhance the sense of immersion for a group of listeners by reproducing the sounds that would originate from several directions around the listeners, thus simulating the way we perceive sound in a real acoustical space. On the other hand, multichannel audio is one of the most demanding media types in terms of transmission requirements. A novel architecture allowing delivery of uncompressed multichannel audio over high-bandwidth communications networks was presented in [1]. As suggested there, for applications in which bandwidth limitations prohibit transmission of multiple audio channels, an alternative would be to transmit only one or two channels (denoted as reference channels or recordings in this work, for example, the left and right signals in a traditional stereo recording) and reconstruct the remaining channels at the receiving end. The system proposed in this paper provides a solution for reconstructing the channels of a specific recording from the reference channels and is particularly suitable for live concert hall performances. The proposed method is based on information of the acoustics of a specific concert hall and the microphone locations with respect to the orchestra; this information can be extracted from the specific multichannel recording.

Before proceeding to the description of the method proposed, a brief outline of the basis of our approach is given. A number of microphones are used to capture several characteristics of the venue, resulting in an equal number of stem recordings (or elements). Figure 1 provides an example of how microphones may be arranged in a recording venue in a multichannel recording. These recordings are then mixed and 


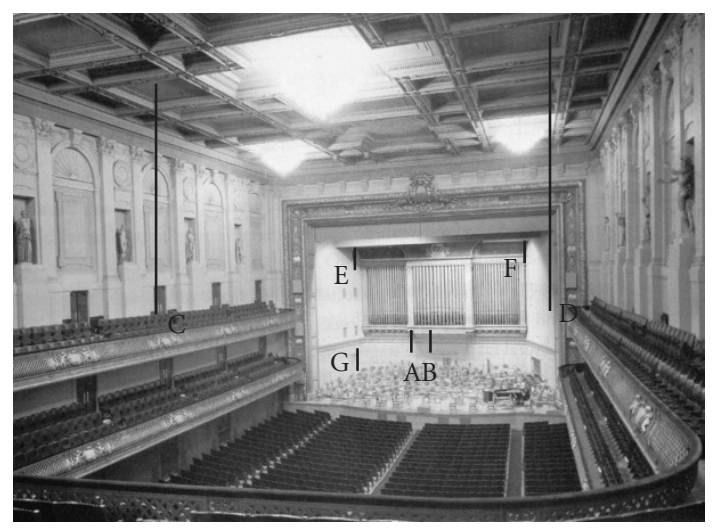

FIgURE 1: An example of how microphones may be arranged in a recording venue for a multichannel recording. In the virtual microphone synthesis algorithm, microphones A and B are the main reference pair from which the remaining microphone signals can be derived. Virtual microphones C and D capture the hall reverberation, while virtual microphones $\mathrm{E}$ and $\mathrm{F}$ capture the reflections from the orchestra stage. Virtual microphone $\mathrm{G}$ can be used to capture individual instruments such as the tympani. These signals can then be mixed and played back through a multichannel audio system that recreates the spatial realism of a large hall.

played back through a multichannel audio system that attempts to recreate the spatial realism of the recording venue. Our objective is to design a system, based on available stem recordings, which is able to recreate all of these recordings from the reference channels at the receiving end (thus, stem recordings are also referred to as target recordings here). The result would be a significant reduction in transmission requirements, while enabling mixing at the receiving end. Consequently, such a system would be suitable for completely resynthesizing any number of channels in the initial recording (i.e., no information about the target recordings needs to be transmitted other than the conversion parameters). This is different than what commercial systems accomplish today. In addition, the system proposed in this paper is a structured representation of multichannel audio that lends itself to other possible applications such as multichannel audio synthesis which is briefly described later in this section. By examining the acoustical characteristics of the various stem recordings, the distinction of microphones is made into reverberant and spot microphones.

Spot microphones are microphones that are placed close to the sound source (e.g., G in Figure 1). These microphones introduce a very challenging situation. Because the source of sound is not a point source but rather distributed such as in an orchestra, the recordings of these microphones depend largely on the instruments that are near the microphone and not so much on the acoustics of the hall. Synthesizing the recordings of these microphones, therefore, involves enhancing certain instruments and diminishing others, which in most cases overlap both in the time and frequency domains. The algorithm described here, focusing on this problem, is based on spectral conversion (SC). The special case of percussive drum-like sounds is separately examined since these sounds are of impulsive nature and cannot be addressed by SC methods. These sounds are of particular interest however since they greatly affect our perception of proximity to the orchestra.

Reverberant microphones are the microphones placed far from the sound source, for example, C and D in Figure 1. These microphones are treated separately as one category because they mainly capture reverberant information (that can be reproduced by the surrounded channels in a multichannel playback system). The recordings captured by these microphones can be synthesized by filtering the reference recordings through linear time-invariant (LTI) filters, designed using the methods that will be described in later sections of this paper. Existing reverberation methods use a combination of comb and all-pass filters to effectively add reverberation to the existing monophonic or stereophonic signal. Our objective is to estimate the appropriate filters that capture the concert hall acoustical properties from a given set of stem microphone recordings. We describe an algorithm that is based on a spectral estimation approach and is particularly suitable for generating such filters for large venues with long reverberation times. Ideally, the resulting filter implements the spectral modification induced by the hall acoustics.

We have obtained such stem microphone recordings from two orchestra halls in the USA by placing microphones at various locations throughout the hall. By recording a performance with a total of sixteen microphones, we then designed a system that recreates these recordings (thus named virtual microphone recordings) from the main microphone pair. It should be noted that the methods proposed here intend to provide a solution for the problem of resynthesizing existing multichannel recordings from a smaller subset of these recordings. The problem of completely synthesizing multichannel recordings from stereophonic (or monophonic) recordings, thus greatly augmenting the listening experience, is not addressed here. The synthesis problem is a topic of related research to appear in a future publication. However, it is important to distinguish the cases where these two problems (synthesis and resynthesis) differ. For reverberant microphones, since the result of our method is a group of LTI filters, both problems are addressed at the same time. The filters designed are capable of recreating the acoustic properties of the venue where the specific recordings took place. If these filters are applied to an arbitrary (nonreverberant) recording, the resulting signal will contain the venue characteristics at the particular microphone location. In such manner, it is possible to completely synthesize reverberant stem recordings and, consequently, a multichannel recording. On the contrary, this will not be possible for the stem microphone methods. As it will be clear later, the algorithms described here are based on the specific recordings that are available. The result is a group of SC functions that are designed by estimating the unknown parameters based on training data that are available from the target recordings. These functions cannot be applied to an arbitrary signal and produce meaningful results. This is an important issue when addressing the synthesis problem and will not be the topic of this paper. 
The remainder of this paper is organized as follows. In Section 2, the spot microphone resynthesis problem is addressed. SC methods are described and applied to the problem in different subbands of the audio signal. The special case of percussive sounds is also examined. In Section 3, the reverberant microphone resynthesis problem is examined. The issue of defining an objective measure of the method performance arises and is addressed by defining a normalized mutual information (NMI) measure. Finally, a brief discussion of the results is given in Section 4 and possible directions for future research on the subject are proposed.

\section{SPOT MICROPHONE RESYNTHESIS}

The methods for spot microphones are geared towards enhancing certain instruments in the reference recording. Note that this problem is different from the source separation problem that seeks to extract the instrument from a signal containing multiple instruments, nor do we attempt to estimate the room impulse response and thus dereverberate the signals. Instead, it is an attempt to simulate what a microphone near a particular instrument would pick up, which includes mostly a "dry" (nonreverberant) version of the instrument and some leakage from nearby instruments. The instruments close to the target microphone are far more prominent in the target recording than in the reference recording. Our objective is to retain the perceptual advantages of the multichannel recording, as a first step towards addressing the problem. This, in effect, means that our objective is to enhance the desired voices/instruments in the reference recording even if the resynthesized signal is not identical with the desired. We were able, as stated later, to produce identical responses for the reverberant microphones case, however the spot microphone case proved to be far more demanding.

For the spot microphones case, nonstationarity of the audio signals is the focus of this paper; the SC methods attempt to address this problem. The problem arises from the fact that the objective of our method is to enhance a particular instrument in the reference recording. The instrument to be enhanced has a frequency response that significantly varies in time, and as a result, a time-invariant filter would not produce meaningful results. Our methods are based on the fact that the reference and target responses are highly related (same performance recorded simultaneously with different microphones). Based on this observation, the desired transfer function, although constantly varying in time, can be estimated, based on the reference recording, with the use of the SC methods. For the spot microphones case, each target microphone captures mainly a specific type of instruments while the reference microphone "weighs" all instruments approximately equally. This corresponds to the dependence of the spot microphones on their location with respect to the orchestra. Although the response of these microphones depends on the acoustics of the hall as well, this dependence is not considered acoustically significant (for reasons explained in Section 2.1), and this greatly simplifies the solution. The methods proposed here result in one conversion function for each pair of spot and reference microphones (with the reference microphone remaining the same in all cases) so that all target waveforms can be resynthesized from only one recording.

\subsection{Spectral conversion}

Our initial experiments for the spot microphones case, detailed in the next paragraph, motivated us to focus on modifying the short-term spectral properties of the reference audio signal in order to recreate the desired one. The shortterm spectral properties are extracted by using a short sliding window with overlapping (resulting in a sequence of signal segments or frames). Each frame is modeled as an autoregressive (AR) filter excited by a residual signal. The AR filter coefficients are found by means of linear predictive (LP) analysis [2] and the residual signal is the result of the inverse filtering of the audio signal of the current frame by the AR filter. The LP coefficients are modified in a way to be described later in this section and the residual is filtered with the designed AR filter to produce the desired signal of the current frame. Finally, the desired response is synthesized from the designed frames using overlap-add techniques [3].

It is interesting to describe one of our initial experiments that led us to focus on the short-term spectral envelope and, as a consequence, on the SC methods that are described next. In this simple experiment, we attempted to synthesize the desired response (in this case the response captured by the microphone is placed close to the chorus of the orchestra) by using the reference residual and the cepstral coefficients obtained from the desired response. In other words, we were interested to test the result of our resynthesis methods in the ideal case where the desired sequence of cepstral coefficients was correctly "predicted." The result was an audio signal which sounded more reverberant than the desired signal (for reasons explained later in this section), but extremely similar in all respects. Thus, deriving an algorithm that correctly predicts the desired sequence of cepstral coefficients from the reference cepstral coefficients of the respective frame would result in a resynthesized signal very close to the desired signal. The problem as stated is exactly the problem statement of SC, which aims to design a mapping function from the reference to the target space, whose parameters remain constant for a particular pair of reference and target sources. The result will be a significant reduction of information as the target response can be reconstructed using the reference signal and this function.

Such a mapping function can be designed by following the approach of voice conversion algorithms $[4,5,6]$. The objective of voice conversion is to modify a speech waveform so that the context remains as it is but appears to be spoken by a specific (target) speaker. Although the application is completely different, the followed approach is very suitable for our problem. In voice conversion, pitch and time scaling need to be considered, while in the application examined here, this is not necessary. This is true since the reference and 
target waveforms come from the same excitation recorded with different microphones and the need is not to modify but to enhance the reference waveform. However, in both cases, there is the need to modify the short-term spectral properties of the waveform.

At this point, it is of interest to mention that the SC methods are useful for modifying the spectral coloration of the signal, and the target response is resynthesized using the modified spectral envelope along with the residual derived from the reference recording. Note that short-term analysis indicates the use of windows in the order of 50 milliseconds, which means that the residual (in effect, the modeling error) contains the reverberation which cannot be modeled with the short-term spectral envelope. As a result, the resynthesized response might sound more reverberant than the target response, depending on how reverberant the reference response originally is. Our concern, though, is mostly to enhance a specific instrument within the reference recording, without focusing on dereverberating the signal. In most cases, this will not be an issue, given that usually the reference recordings are not highly reverberant.

Assuming that a sequence $\left[\mathbf{x}_{1} \mathbf{x}_{2} \cdots \mathbf{x}_{n}\right]$ of reference spectral vectors (e.g., line spectral frequencies (LSFs), cepstral coefficients, etc.) is given, as well as the corresponding sequence of target spectral vectors $\left[\mathbf{y}_{1} \mathbf{y}_{2} \cdots \mathbf{y}_{n}\right]$ (training data from the reference and target recordings, respectively), a function $\mathscr{F}(\cdot)$ can be designed which, when applied to vector $\mathbf{x}_{k}$, produces a vector close in some sense to vector $\mathbf{y}_{k}$. Many algorithms have been described for designing this function (see $[4,5,6,7]$ and the references therein). Here the algorithms based on vector quantization (VQ) [4] and Gaussian mixture models (GMM) $[5,6]$ were implemented and compared.

\subsubsection{SC based on VQ}

Under this approach, the spectral vectors of the reference and target signals (training data) are vector quantized using the well-known modified $K$-means clustering algorithm (see, e.g., [8] for details). Then, a histogram is created indicating the correspondences between the reference and target centroids. Finally, the function $\mathscr{F}$ is defined as the linear combination of the target centroids using the designed histogram as a weighting function. It is important to mention that in this case the spectral vectors were chosen to be the cepstral coefficients so that the distance measure used in clustering is the truncated cepstral distance.

\subsubsection{SC based on GMM}

In this case, the assumption made is that the sequence of spectral vectors $\mathbf{x}_{k}$ is a realization of a random vector $\mathbf{x}$ with a probability density function (pdf) that can be modeled as a mixture of $M$ multivariate Gaussian pdfs. GMMs have been repeatedly used in such manner to model the properties of audio signals with reasonable success (see, e.g., $[9,10,11]$ ).

According to GMMs, the pdf of $\mathbf{x}, \mathrm{g}(\mathbf{x})$, can be written as

$$
\mathrm{g}(\mathbf{x})=\sum_{i=1}^{M} p\left(\omega_{i}\right) \mathcal{N}\left(\mathbf{x} ; \boldsymbol{\mu}_{i}^{x}, \boldsymbol{\Sigma}_{i}^{x x}\right),
$$

where $\mathcal{N}(\mathbf{x} ; \boldsymbol{\mu}, \boldsymbol{\Sigma})$ is the normal multivariate distribution with mean vector $\boldsymbol{\mu}$ and covariance matrix $\boldsymbol{\Sigma}$, and $p\left(\omega_{i}\right)$ is the prior probability of class $\omega_{i}$. The parameters of the GMM, that is, the mean vectors, covariance matrices, and priors, can be estimated using the expectation maximization (EM) algorithm [12].

As already mentioned, the function $\mathscr{F}$ is designed so that the spectral vectors $\mathbf{y}_{k}$ and $\mathscr{F}\left(\mathbf{x}_{k}\right)$ are close in some sense. In [5], the function $\mathscr{F}$ is designed such that the error

$$
\mathscr{E}=\sum_{k=1}^{n}\left\|\mathbf{y}_{k}-\mathscr{F}\left(\mathbf{x}_{k}\right)\right\|^{2}
$$

is minimized. Since this method is based on least squares estimation, it will be denoted as the LSE method. This problem becomes possible to solve under the constraint that $\mathscr{F}_{F}$ is piecewise linear, that is,

$$
\mathscr{F}\left(\mathbf{x}_{k}\right)=\sum_{i=1}^{M} p\left(\omega_{i} \mid \mathbf{x}_{k}\right)\left[\mathbf{v}_{i}+\boldsymbol{\Gamma}_{i} \boldsymbol{\Sigma}_{i}^{x x^{-1}}\left(\mathbf{x}_{k}-\boldsymbol{\mu}_{i}^{x}\right)\right],
$$

where the conditional probability that a given vector $\mathbf{x}_{k}$ belongs to class $\omega_{i}, p\left(\omega_{i} \mid \mathbf{x}_{k}\right)$, can be computed by applying Bayes' theorem:

$$
p\left(\omega_{i} \mid \mathbf{x}_{k}\right)=\frac{p\left(\omega_{i}\right) \mathcal{N}\left(\mathbf{x}_{k} ; \boldsymbol{\mu}_{i}^{x}, \boldsymbol{\Sigma}_{i}^{x x}\right)}{\sum_{j=1}^{M} p\left(\omega_{j}\right) \mathcal{N}\left(\mathbf{x}_{k} ; \boldsymbol{\mu}_{j}^{x}, \boldsymbol{\Sigma}_{j}^{x x}\right)} .
$$

The unknown parameters $\left(\mathbf{v}_{i}\right.$ and $\left.\boldsymbol{\Gamma}_{i}, i=1, \ldots, M\right)$ can be found by minimizing (2) which reduces to solving a typical least squares equation.

A different solution for function $\mathscr{F}$ results when a different function than (2) is minimized [6]. Assuming that $\mathbf{x}$ and $\mathbf{y}$ are jointly Gaussian for each class $\omega_{i}$, then, in meansquared sense, the optimal choice for the function $\mathscr{F}$ is

$$
\begin{aligned}
\mathscr{F}\left(\mathbf{x}_{k}\right) & =\mathrm{E}\left(\mathbf{y} \mid \mathbf{x}_{k}\right) \\
& =\sum_{i=1}^{M} p\left(\omega_{i} \mid \mathbf{x}_{k}\right)\left[\boldsymbol{\mu}_{i}^{y}+\boldsymbol{\Sigma}_{i}^{y x} \boldsymbol{\Sigma}_{i}^{x x^{-1}}\left(\mathbf{x}_{k}-\boldsymbol{\mu}_{i}^{x}\right)\right],
\end{aligned}
$$

where $\mathrm{E}(\cdot)$ denotes the expectation operator and the conditional probabilities $p\left(\omega_{i} \mid \mathbf{x}_{k}\right)$ are given again from (4). If the source and target vectors are concatenated, creating a new sequence of vectors $\mathbf{z}_{k}$ that are the realizations of the random vector $\mathbf{z}=\left[\mathbf{x}^{T} \mathbf{y}^{T}\right]^{T}$ (where ${ }^{T}$ denotes transposition), then all the required parameters in the above equations can be found by estimating the GMM parameters of $\mathbf{z}$. Then,

$$
\boldsymbol{\Sigma}_{i}^{z z}=\left[\begin{array}{cc}
\boldsymbol{\Sigma}_{i}^{x x} & \boldsymbol{\Sigma}_{i}^{x y} \\
\boldsymbol{\Sigma}_{i}^{y x} & \boldsymbol{\Sigma}_{i}^{y y}
\end{array}\right], \quad \boldsymbol{\mu}_{i}^{z}=\left[\begin{array}{c}
\boldsymbol{\mu}_{i}^{x} \\
\boldsymbol{\mu}_{i}^{y}
\end{array}\right] .
$$

Once again, these parameters are estimated by the EM algorithm. Since this method estimates the desired function based on the joint density of $\mathbf{x}$ and $\mathbf{y}$, it will be referred to as the joint density estimation (JDE) method. 


\subsection{Subband processing}

Audio signals contain information about a larger bandwidth than speech signals. The sampling rate for audio signals is usually 44.1 or $48 \mathrm{kHz}$ compared with $16 \mathrm{kHz}$ for speech. Moreover, since high acoustical quality for audio is essential, it is important to consider the entire spectrum in detail. For these reasons, the decision to follow an analysis in subbands seems natural. Instead of warping the frequency spectrum using the Bark scale as is usual in speech analysis, the frequency spectrum was divided in subbands and each one was treated separately under the analysis presented in the previous section (the signals were demodulated and decimated after they were passed through the filter banks and before the linear predictive analysis). Perfect reconstruction filter banks, based on wavelets [13], provide a solution with acceptable computational complexity as well as the appropriate, for audio signals, octave frequency division. The choice of filter bank was not a subject of investigation but steep transition from passband to stopband is desirable. The reason is that the short-term spectral envelope is modified separately for each band, thus frequency overlapping between adjacent subbands would result in a distorted synthesized signal.

\subsection{Residual processing for percussive sounds}

The SC methods described earlier will not produce the desired result in all cases. Transient sounds cannot be adequately processed by altering their spectral envelope and must be examined separately. An example of an analysis/synthesis model that treats transient sounds separately and is very suitable as an alternative to the subband-based residual/LP model that we employed is described in [14]. It is suitable since it also models the audio signal in different bands, in each one as a sinusoidal/residual model $[15,16]$. The sinusoidal parameters can be treated in the same manner as the LP coefficients during SC [17]. We are currently considering this model for improving the produced sound quality of our system. However, no structured model is proposed in [14] for transient sounds. In the remainder of this section, the special case of percussive sounds is addressed.

The case of percussive drum-like sounds is considered of particular importance. It is usual in multichannel recordings to place a microphone close to the tympani as drumlike sounds are considered perceptually important in recreating the acoustical environment of the recording venue. For percussive sounds, a similar model to the residual/LP model described here can be used [18] (see also [19, 20, 21]), but for the enhancement purposes investigated in this paper, the emphasis is given to the residual instead of the LP parameters. The idea is to extract the residual of an instance of the particular percussive instrument from the recording of the microphone that captures this instrument and then recreate this channel from the reference channel by simply substituting the residual of all instances of this instrument with the extracted residual. As explained in [18], this residual corresponds to the interaction between the exciter and the resonating body of the instrument and lasts until the structure reaches a steady vibration. This signal characterizes the attack part of the sound and is independent of the frequencies and amplitudes of the harmonics of the produced sound (after the instrument has reached a steady vibration). Thus, it can be used for synthesizing different sounds by using an appropriate all-pole filter. This method proved to be quite successful and further details are given in Section 2.4. The drawback of this approach is that a robust algorithm is required for identifying the particular instrument instances in the reference recording. A possible improvement of the proposed method would be to extract all instances of the instrument from the target response and use some clustering technique for choosing the residual that is more appropriate in the resynthesis stage. The reason is that the residual/LP model introduces modeling error which is larger in the spectral valleys of the AR spectrum; thus, better results would be obtained by using a residual which corresponds to an AR filter as close as possible to the resynthesis AR filter. However, this approach would again require robustly identifying all the instances of the instrument.

\subsection{Implementation details}

The three SC methods outlined in Section 2.1 were implemented and tested using a multichannel recording, obtained as described in Section 1. The objective was to recreate the channel that mainly captured the chorus of the orchestra (residual processing for percussive sound resynthesis is also considered at the last paragraph of this section). Acoustically, therefore, the emphasis was on the male and female voices. At the same time, it was clear that some instruments, inaudible in the target recording but particularly audible in the reference recording, are needed to be attenuated. More generally, it might hold that a spot microphone might enhance more than one type of musical sources. Usually, such microphones are placed with a particular type of instruments in mind, which is easy to discern by acoustical examination, but, in general, careful selection of the training data will result in the desirable result even in complex cases.

A database of about 10000 spectral vectors for each band was created so that only parts of the recording where the chorus is present are used, with the choice of spectral vectors being the cepstral coefficients. Parts of the chorus recording were selected so that there were no segments of silence included. Given that our focus was on modifying the shortterm spectral properties of the reference signal, the analysis window we used was a 2048 sample window for a $44.1 \mathrm{kHz}$ sampling rate. This is a typical value often used when the objective is to alter the short-term spectral properties of audio signals, and was found to produce good sound quality results in our case as well. Results were evaluated through informal listening tests and through objective performance criteria. The SC methods were found to provide promising enhancement results. The experimental conditions are given in Table 1. The number of octave bands used was eight, a choice that gives particular emphasis on the frequency band $0-5 \mathrm{kHz}$ and at the same time does not impose excessive computational demands. The frequency range $0-5 \mathrm{kHz}$ is 
TABle 1: Parameters for the chorus microphone example.

\begin{tabular}{l|rr|c|c}
\hline \multirow{3}{*}{$\begin{array}{l}\text { Band } \\
\text { no. }\end{array}$} & \multicolumn{2}{|c|}{ Frequency range } & LP order & $\begin{array}{l}\text { GMM } \\
\text { centroids }\end{array}$ \\
\cline { 2 - 3 } & Low $(\mathrm{kHz})$ & High $(\mathrm{kHz})$ & & 4 \\
2 & 0.0000 & 0.1723 & 4 & 4 \\
3 & 0.1723 & 0.3446 & 4 & 8 \\
4 & 0.3446 & 0.6891 & 8 & 16 \\
5 & 0.6891 & 1.3782 & 16 & 16 \\
6 & 1.3782 & 2.7563 & 32 & 16 \\
7 & 2.7563 & 5.5125 & 32 & 16 \\
8 & 5.5125 & 11.0250 & 32 & 16 \\
\hline
\end{tabular}

TABLE 2: Normalized distances for LSE-, JDE-, and VQ-based methods.

\begin{tabular}{l|cc|c}
\hline \multirow{2}{*}{$\begin{array}{l}\text { SC } \\
\text { method }\end{array}$} & \multicolumn{2}{|c|}{ Cepstral distance } & \multirow{2}{*}{$\begin{array}{l}\text { Centroids } \\
\text { per band }\end{array}$} \\
\cline { 2 - 3 } & Train & Test & Table 1 \\
LSE & 0.6451 & 0.7144 & Table 1 \\
JDE & 0.6629 & 0.7445 & 1024 \\
VQ & 1.2903 & 1.3338 & \\
\hline
\end{tabular}

particularly important for the specific case of chorus recording resynthesis since this is the frequency range where the human voice is mostly concentrated. For producing better results, the entire frequency range $0-20 \mathrm{kHz}$ must be considered. The order of the LP filter varied depending on the frequency detail of each band, and for the same reason, the number of centroids for each band was different.

In Table 2, the average quadratic cepstral distance (averaged over all vectors and all eight bands) is given for each method, for the training data as well as for the data used for testing (nine seconds of music from the same recording). The cepstral distance is normalized with the average quadratic distance between the reference and the target waveforms (i.e., without any conversion of the LP parameters). The improvement is large for both the GMM-based algorithms, with the LSE algorithm being slightly better, and for both the training and testing data. The VQ-based algorithm, in contrast, produced a deterioration in performance which was audible as well. This can be explained based on the fact that the GMMbased methods result in a conversion function which is continuous with respect to the spectral vectors. The VQ-based method, on the other hand, produces audible artifacts introduced by spectral discontinuities because the conversion is based on a limited number of existing spectral vectors. This is the reason why a large number of centroids was used for the VQ-based algorithm as seen in Table 2 compared with the number of centroids used for the GMM-based algorithms. However, the results for the VQ-based algorithm were still unacceptable from both the objective and subjective perspectives (a higher number of centroids was tested, up to 8192 , without any significant improvement).

The algorithm described in Section 2.1 considering the special case of percussive sound resynthesis was tested as well.

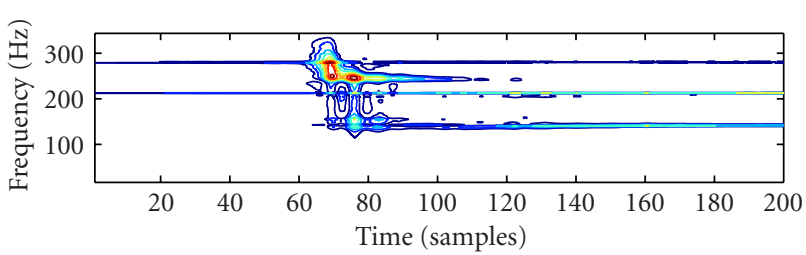

(a)

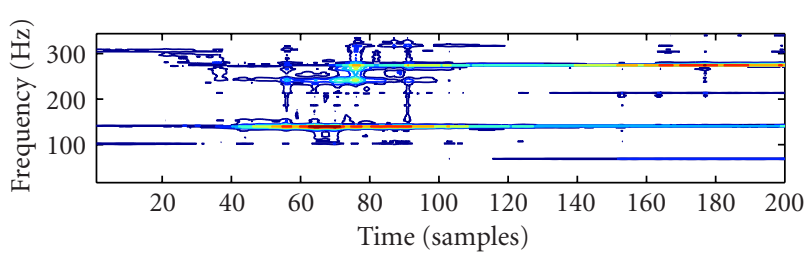

(b)

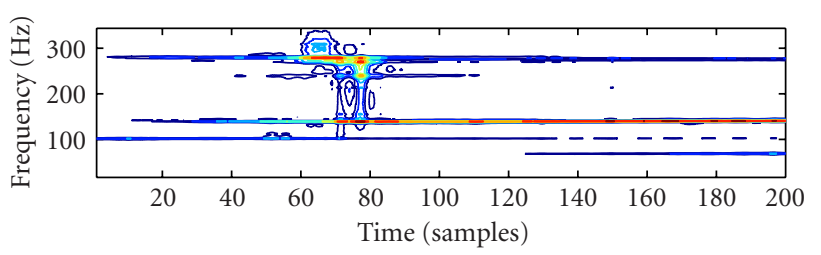

(c)

FIgure 2: Choi-Williams distribution of the desired (a), reference (b), and synthesized (c) waveforms at the time points during a tympani strike (60-80 samples).

Figure 2 shows the time-frequency evolution of a tympani instance using the Choi-Williams distribution [22], a distribution that achieves the high resolution needed in such cases of impulsive signal nature. Figure 2 clearly demonstrates the improvement in drum-like sound resynthesis. The impulsiveness of the signal at around samples $60-80$ is observed in the desired response and verified in the synthesized waveform. The attack part is clearly enhanced, significantly adding naturalness in the audio signal, as our informal listening tests clearly demonstrated.

The methods described in this section can be used for synthesizing recordings of microphones that are placed close to the orchestra. Of importance in this case were the shortterm spectral properties of the audio signals. Thus, LTI filters were not suitable and the time-frequency properties of the waveforms had to be exploited in order to obtain a solution. In Section 3, we focus on microphones placed far from the orchestra and thus containing mainly reverberant signals. As we demonstrate, the desired waveforms can be synthesized by taking advantage of the long-term spectral properties of the reference and the desired signals.

\section{REVERBERANT MICROPHONE SIGNAL SYNTHESIS}

The problem of synthesizing a virtual microphone signal from a signal recorded at a different position in the room can 
be described as follows. Given two processes $s_{1}$ and $s_{2}$, determine the optimal filter $H$ that can be applied to $s_{1}$ (the reference microphone signal) so that the resulting process $s_{2}^{\prime}$ (the virtual microphone signal) is as close as possible to $s_{2}$. The optimality of the resulting filter $H$ is based on how "close" $s_{2}^{\prime}$ is to $s_{2}$. For the case of audio signals, the distance between these two processes must be measured in a way that is psychoacoustically valid. For microphones placed far from the orchestra (reverberant microphones), the main factor that differentiates the target from the reference recording is hall reverberation; thus, in this case, the transfer function is inherently time invariant. This is a typical problem of identification, however in our case we estimate the room response based on existing recordings since it would be impractical or even impossible to measure the hall response for every different recording. At the same time, the nonstationarity of the audio signals that might prevent us from accurate estimation of the transfer functions is addressed by the spectral estimation methods explained in Section 3.1. Another important issue that arises is the fact that the physical system is characterized by a long impulse response. For a typical large symphony hall, the reverberation time is approximately two seconds, which would require a filter of more than 96000 taps to describe the reverberation process (for a typical sampling rate of $48 \mathrm{kHz}$ ). This issue consequently affects both the filter design and the system implementation. While the filter design problem is appropriately addressed, the resulting filters are of inevitably high order, prohibiting cost-effective realtime applications of our methods.

For the reverberant microphones case, the orchestra is considered as a point source. For all practical purposes, this is a valid assumption to make. The distant microphones are not trying to recreate the physical sound field generated by a complex sound source such as the orchestra. Rather, they are trying to provide us with a signal that can be combined with signals from other microphones (real and synthesized) using aesthetic (not mathematical) rules for mixing into a multichannel performance. It is well known that trying to use microphones to capture the physical sound waves at one point in space is not physically possible and does not correspond to the way a human listener would hear/perceive it even if it were. As explained later in this section, our listening tests indicate that the assumption made is a valid one, with the target and resynthesized waveforms acoustically indistinguishable (for appropriate filter orders).

\subsection{IIR filter design}

There are several possible approaches to the problem. One is to use classical estimation theoretic techniques such as least squares or Wiener filtering-based algorithms to estimate the hall environment with a long finite-duration impulse response (FIR) or infinite-duration impulse response (IIR) filter. Adaptive algorithms such as LMS [2] can provide an acceptable solution in such system identification problems while least squares methods suffer prohibitive computational demands. For LMS, the limitation lies in the fact that the input and the output are nonstationary signals making convergence quite slow. In addition, the required length of the filter is very large, so such algorithms would prove to be inefficient for this problem. Although it is possible to prewhiten the input of the adaptive algorithm (see, e.g., $[2,23]$ and the references therein) so that convergence is improved, these algorithms still have not proved to be efficient for this problem.

An alternative to the aforementioned methods for treating system identification problems is to use spectral estimation techniques based on the cross spectrum [24]. These methods are divided into parametric and nonparametric. Nonparametric methods based on averaging techniques such as the averaged periodogram (Welch spectral estimate) [25, $26,27]$ are considered more appropriate for the case of long observations and for nonstationary conditions since no model is assumed for the observed data (a different approach based on the cross spectrum which, instead of averaging, solves an overdetermined system of equations can be found in [28]). After the frequency response of the filter is estimated, an IIR filter can be designed based on that response. The advantage of this approach is that IIR filters are a more natural choice of modeling the physical system under consideration and can be expected to be very efficient in approximating the spectral properties of the recording venue. In addition, an IIR filter would implement the desired frequency response with a significantly lower order compared with an FIR filter. Caution must, of course, be taken in order to ensure the stability of the filters.

To summarize, if we could define a power spectral density $S_{s_{1}}(\omega)$ for signal $s_{1}$ and $S_{s_{2}}(\omega)$ for signal $s_{2}$, then it would be possible to design filter $H(\omega)$ that can be applied to process $s_{1}$ resulting in process $s_{2}^{\prime}$, which is intended to be an estimate of $s_{2}$. The filter $H(\omega)$ can be estimated by means of spectral estimation techniques. Furthermore, if $S_{s_{1}}(\omega)$ is modeled by an all-pole approximation $\left|1 / A_{p 1}\right|^{2}$ and $S_{s_{2}}(\omega)$ similarly as $\left|1 / A_{p 2}\right|^{2}$, then $H=A_{p 1} / A_{p 2}$ if $H$ is restricted to be the minimum phase spectral factor of $|H(\omega)|^{2}$. The result is a minimum-phase stable IIR filter that can be efficiently designed. The analysis that follows provides the details for designing $H$.

The estimation of $H(\omega)$ is based on computing the cross spectrum $S_{s_{2} s_{1}}$ of signals $s_{2}$ and $s_{1}$ and the autospectrum $S_{s_{1}}$ of signal $s_{1}$. It is true that if these signals were stationary, then

$$
S_{s_{2} s_{1}}(\omega)=H(\omega) S_{s_{1}}(\omega) .
$$

The difficulties arising in the design of filter $H$ are due to the nonstationary nature of audio signals. This issue can be partly addressed if the signals are divided into segments short enough to be considered of approximately stationary nature. It must be noted, however, that these segments must be large enough so that they can be considered long compared with the length of the impulse response that must be estimated in order to avoid edge effects (as explained in [29], where a similar procedure is followed for the case of blind deconvolution for audio signal restoration). 
For interval $i$, composed of $M$ (real) samples $s_{1}^{(i)}(0)$, $\ldots, s_{1}^{(i)}(M-1)$, the empirical transfer function estimate (ETFE) [24] is computed as

$$
\hat{H}^{(i)}(\omega)=\frac{S_{2}^{(i)}(\omega)}{S_{1}^{(i)}(\omega)},
$$

where

$$
S_{1}^{(i)}(\omega)=\sum_{n=0}^{M-1} s_{1}^{(i)}(n) e^{-j \omega n}
$$

is the Fourier transform of the segment samples, though this cannot be considered an accurate estimate of $H(\omega)$, since the filter $H^{(i)}(\omega)$ will be valid only for frequencies corresponding to the harmonics of segment $i$ (under the valid assumption of quasiperiodic nature of the audio signal for each segment). An intuitive procedure would be to obtain the estimate of the spectral properties of the recording venue $\hat{H}(\omega)$ by averaging all the estimates available. Since the ETFE is the result of frequency division, it is apparent that in frequencies where $S_{s_{1}}(\omega)$ is close to zero, the ETFE would become unstable, so a more robust procedure would be to estimate $H$ using a weighted average of the $K$ segments available [24], that is,

$$
\hat{H}(\omega)=\frac{\sum_{i=0}^{K-1} \beta^{(i)}(\omega) H^{(i)}(\omega)}{\sum_{i=0}^{K-1} \beta^{(i)}(\omega)} .
$$

A sensible choice of weights would be

$$
\beta^{(i)}(\omega)=\left|S_{1}^{(i)}(\omega)\right|^{2} .
$$

It can be easily shown that estimating $H$ under this approach is equivalent to estimating the autospectrum of $s_{1}$ and the cross spectrum of $s_{2}$ and $s_{1}$ using the Cooley-Tukey spectral estimate [26] (in essence, Welch spectral estimation with rectangular windowing of the data and no overlapping). In other words, defining the power spectrum estimate under the Cooley-Tukey procedure as

$$
S_{s_{1}}^{C T}(\omega)=\frac{1}{K} \sum_{i=0}^{K-1}\left|S_{1}^{(i)}(\omega)\right|^{2}
$$

where $S(\omega)$ is defined as previously, and a similar expression for the cross spectrum

$$
S_{S_{2} S_{1}}^{C T}(\omega)=\frac{1}{K} \sum_{i=0}^{K-1} S_{2}^{(i)}(\omega) S_{1}^{(i) *}(\omega),
$$

it holds that

$$
\hat{H}(\omega)=\frac{S_{s_{2} s_{1}}^{C T}(\omega)}{S_{s_{1}}^{C T}(\omega)}
$$

which is analogous to (7). Thus, for a stationary signal, the averaging of the estimated filters is justifiable. A window can additionally be used to further smooth the spectra.
The described method is meaningful for the special case of audio signals despite their nonstationarity. It is well known that the averaged periodogram provides a smoothed version of the periodogram. Considering that it is true even for nonstationary (but of finite length) signals that

$$
S_{2}(\omega) S_{1}^{*}(\omega)=H(\omega)\left|S_{1}(\omega)\right|^{2},
$$

then averaging in essence smoothes the frequency response of $H$. This is justifiable since it is true that a nonsmoothed $H$ will contain details that are of no acoustical significance. Further smoothing can yield a lower-order IIR filter by taking advantage of AR modeling. Considering signal $s_{1}$, the inverse Fourier transform of its power spectrum $S_{s_{1}}(\omega)$, derived as described earlier, will yield the sequence $r_{s_{1}}(m)$. If this sequence is viewed as the autocorrelation of $s_{1}$ and the samples $r_{s_{1}}(0), \ldots, r_{s_{1}}(p+1)$ are inserted in the Wiener-Hopf equations for linear prediction (with the AR order $p$ being significantly smaller than the number of samples of each block $M$ for smoothing the spectra):

$$
\begin{gathered}
{\left[\begin{array}{cccc}
r_{s_{1}}(0) & r_{s_{1}}(1) & \cdots & r_{s_{1}}(p-1) \\
r_{s_{1}}(1) & r_{s_{1}}(0) & \cdots & r_{s_{1}}(p-2) \\
\vdots & \vdots & \ddots & \vdots \\
r_{s_{1}}(p-1) & r_{s_{1}}(p-2) & \cdots & r_{s_{1}}(0)
\end{array}\right]\left[\begin{array}{c}
a_{p 1}(1) \\
a_{p 1}(2) \\
\vdots \\
a_{p 1}(p)
\end{array}\right]} \\
=\left[\begin{array}{c}
r_{s_{1}}(1) \\
r_{s_{1}}(2) \\
\vdots \\
r_{s_{1}}(p)
\end{array}\right],
\end{gathered}
$$

then the coefficients $a_{p 1}(i)$ result in an approximation of $S_{s_{1}}(\omega)$ (omitting the constant gain term which is not of importance in this case):

$$
S_{S_{1}}(\omega)=\left|\frac{1}{A_{p 1}(\omega)}\right|^{2},
$$

where

$$
A_{p 1}(\omega)=1+\sum_{l=1}^{p} a_{p 1}(l) e^{-j \omega l} .
$$

A similar expression holds for $S_{s_{2}}(\omega)$. The spectra $S_{s_{1}}$ and $S_{s_{2}}$ can be computed as in (12). Using the fact that

$$
S_{s_{2}}(\omega)=|H(\omega)|^{2} S_{s_{1}}(\omega)
$$

and restricting $H$ to be minimum phase, we find from the spectral factorization of (19) that a solution for $H$ is

$$
H(\omega)=\frac{A_{p 1}(\omega)}{A_{p 2}(\omega)} .
$$

Filter $H$ can be designed very efficiently even for very large filter orders following this method since (16) can be solved using the Levinson-Durbin recursion. This filter will be IIR and stable. 
A problem with the aforementioned design method is that the filter $H$ is restricted to be of minimum phase. It is of interest to mention that in our experiments the minimum phase assumption proved to be perceptually acceptable. This can be possibly attributed to the fact that if the minimum phase filter $H$ captures a significant part of the hall reverberation, then the listener's ear will be less sensitive to the phase distortion [30]. It is not possible, however, to generalize this observation and the performance of this last step in the filter design will possibly vary depending on the particular characteristics of the venue captured in the multichannel recording.

\subsection{Mutual information as a spectral distortion measure}

As previously mentioned, we need to apply the above procedure in blocks of data of the two processes $s_{1}$ and $s_{2}$. In our experiments, we chose signal block lengths of 100000 samples (long blocks of data are required due to the long of reverberation time of the hall as explained earlier). We then experimented with various orders of filters $A_{p 1}$ and $A_{p 2}$. As expected, relatively high orders were required to reproduce $s_{2}$ from $s_{1}$ with an acceptable error between $s_{2}^{\prime}$ (the resynthesized process) and $s_{2}$ (the target recording). The performance was assessed through blind $\mathrm{A} / \mathrm{B} / \mathrm{X}$ listening evaluation. An order of 10000 coefficients for both the numerator and denominator of $H$ resulted in an error between the original and synthesized signals that was not detectable by listeners. We also evaluated the performance of the filter by synthesizing blocks from a part of the signal other than the one that was used for designing the filter. Again, the A/B/X evaluation showed that for orders higher than 10000 , the synthesized signal was indistinguishable from the original. Although such high-order filters are impractical for real-time applications, the performance of our method is an indication that the model is valid, therefore motivating us to further investigate filter optimization. This method can be used for offline applications such as remastering old recordings and requiring a reasonable amount of time for resynthesis that depends on the specific platform and implementation. A real-time version was also implemented using the Lake DSP Huron digital audio convolution workstation. With this system, we are able to synthesize 12 virtual microphone stem recordings from a monophonic or stereophonic compact disc (CD) in real time. It is interesting to mention that our informal listening tests showed that for filter orders of 5000 or less, the amount of reverberation perceived in the signal is not sufficient. This is not surprising, given the physical size (150' in length) and reverberation characteristics ( 1.9 seconds) of the hall in which we conducted our experiments.

To obtain an objective measure of the performance, it is necessary to derive a mathematical measure of the distance between the synthesized and the original processes. The difficulty in defining such a measure is that it must also be psychoacoustically valid. This problem has been addressed in speech processing where measures such as the log spectral distance and the Itakura-Saito distance are used [31]. In our case, we need to compare the spectral characteristics of

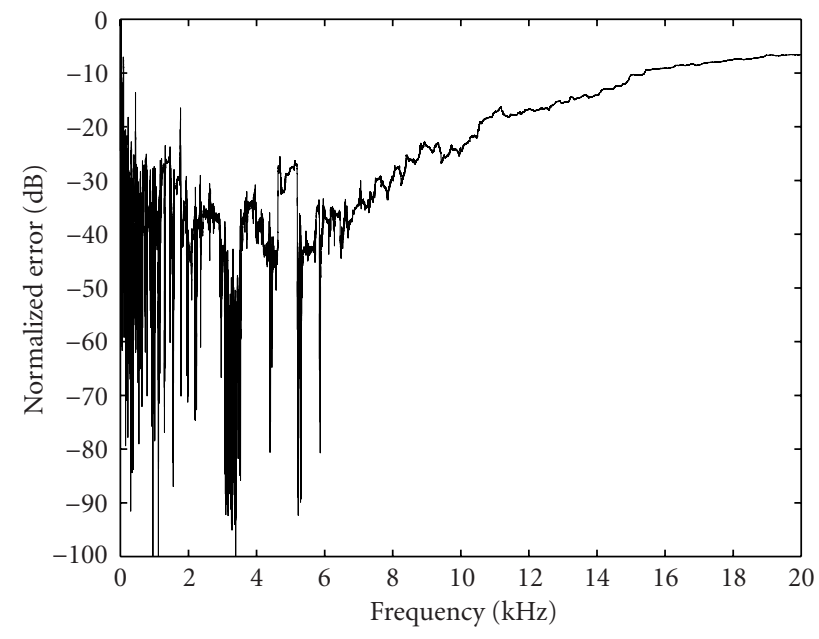

FIGURE 3: Normalized error between original and synthesized microphone signals as a function of frequency.

long sequences with spectra that contain a large number of peaks and dips that are narrow enough to be imperceptible to the human ear. In other words, the focus is on the longterm spectral properties of the audio signals, while spectral distortion measures have been developed for comparing the short-term spectral properties of signals. To overcome comparison inaccuracies that would be mathematical rather than psychoacoustical in nature, we chose to perform 1/3 octave smoothing [32] and compare the resulting smoothed spectral cues. The results are shown in Figure 3 in which we compare the spectra of the original (measured) microphone signal and the synthesized signal. The two spectra are practically indistinguishable below $10 \mathrm{kHz}$. Although the error increases at higher frequencies, the listening evaluations show that this is not perceptually significant. One problem that was encountered while comparing the $1 / 3$ octave smoothed spectra was the fact that the average error was not reduced with increasing filter order as rapidly as the results of the listening tests suggested. To address this inconsistency, we experimented with various distortion measures.

These measures included the root mean square (RMS) log spectral distance, the truncated cepstral distance, and the Itakura distance (for a description of all these measures, see, e.g., [8]). The results, however, were still not inline with what the listening evaluations indicated. This led us to a measure that is commonly used in pattern comparison and is known as the mutual information (see, e.g., [33]). By definition, the mutual information of two random variables $X$ and $Y$ with joint pdf $p(x, y)$ and marginal pdfs $p(x)$ and $p(y)$ is the relative entropy between the joint distribution and the product distribution, that is,

$$
I(X ; Y)=\sum_{x \in \mathscr{X}} \sum_{y \in \mathscr{Y}} p(x, y) \log \frac{p(x, y)}{p(x) p(y)} .
$$

It is easy to prove that

$$
I(X ; Y)=H(X)-H(X \mid Y)=H(Y)-H(Y \mid X)
$$


and also

$$
I(X ; Y)=H(X)+H(Y)-H(X, Y),
$$

where $H(X)$ is the entropy of $X$,

$$
H(X)=-\sum_{x \in \mathscr{X}} p(x) \log p(x) .
$$

Similarly, $H(Y)$ is the entropy of $Y$. The term $H(X \mid Y)$ is the conditional entropy defined as

$$
\begin{aligned}
H(X \mid Y) & =\sum_{y \in \mathcal{O}} p(y) H(X \mid Y=y) \\
& =-\sum_{y \in \mathscr{Y}} p(y) \sum_{x \in \mathscr{X}} p(x \mid y) \log p(x \mid y)
\end{aligned}
$$

while $H(X, Y)$ is the joint entropy defined as

$$
H(X, Y)=-\sum_{x \in \mathscr{X}} \sum_{y \in \mathscr{Y}} p(x, y) \log p(x, y) .
$$

The mutual information is always positive. Since our interest is in comparing two vectors $X$ and $Y$ with $Y$ being the desired response, it is useful to use a modified definition for the mutual information, the NMI $I_{N}(X ; Y)$, which can be defined as

$$
I_{N}(X ; Y)=\frac{H(Y)-H(Y \mid X)}{H(Y)}=\frac{I(X ; Y)}{H(Y)} .
$$

This version of the mutual information is mentioned in [33, page 47] and has been applied in many applications as an optimization measure (e.g., radar remote sensing applications [34]). Obviously,

$$
0 \leq I_{N}(X ; Y) \leq 1 .
$$

The NMI obtains its minimum value when $X$ and $Y$ are statistically independent and its maximum value when $X=Y$. The NMI does not constitute a metric since it lacks symmetry. On the other hand, the NMI is invariant to amplitude differences [35], which is a very important property, especially for comparing audio waveforms.

The spectra of the original and the synthesized responses were compared using the NMI for various filter orders and the results are depicted in Figure 4. The NMI increases with filter order both when considering the raw spectra and when using the spectra that were smoothed using AR modeling (spectral envelope by all-pole modeling with linear predictive coefficients). We believe that the calculated NMI using the smoothed spectra is the measure that closely approximates the results we achieved from the listening tests. As can be seen from the figure, the NMI for a filter order of 20000 is 0.9386 (i.e., close to unity which corresponds to indistinguishable similarity) for the LP spectra while the NMI for the same order but for the raw spectra is 0.5124 . Furthermore, the fact that both the raw and smoothed NMI measures increase monotonically in the same fashion indicates that the

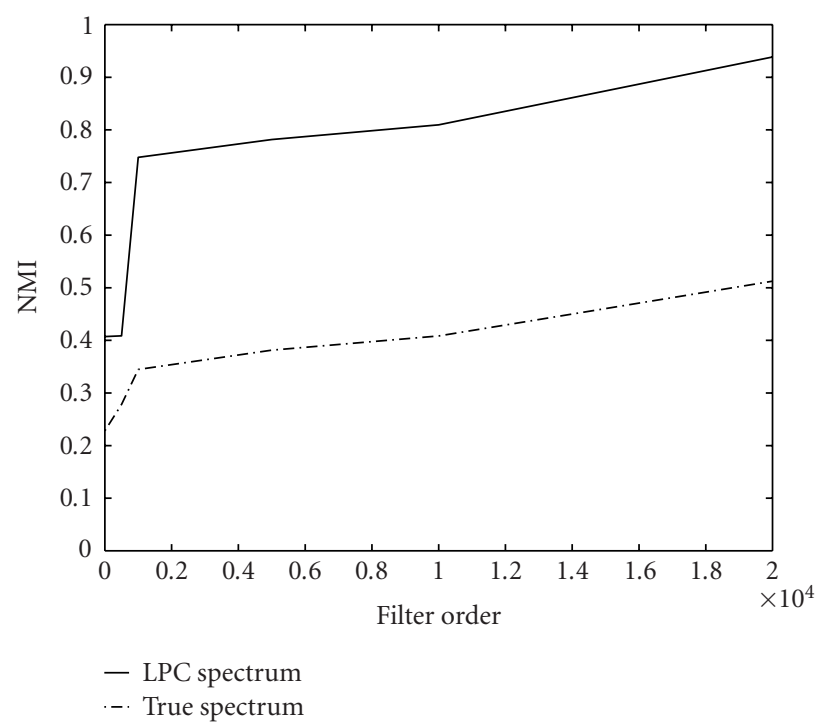

FIGURE 4: NMI between original and synthesized microphone signals as a function of filter order.

smoothing is valid since it only reduces the "distance" between the two waveforms in a proportionate way for all the synthesized waveforms (order 0 in the diagram corresponds to no filtering; it is the distance between the original and the reference waveforms).

\section{CONCLUSIONS AND FUTURE RESEARCH}

Multichannel audio resynthesis is a new and important application that allows transmission of only one or two channels of multichannel audio and resynthesis of the remaining channels at the receiving end. It offers the advantage that the stem microphone recordings can be resynthesized at the receiving end, which makes this system suitable for many professional applications and, at the same time, poses no restrictions on the number of channels of the initial multichannel recording. The distinction was made of the methods employed, depending on the location of the "virtual" microphones, namely, spot and reverberant microphones. Reverberant microphones are those that are placed at some distance from the sound source (e.g., the orchestra) and therefore, contain more reverberation. On the other hand, spot microphones are located close to individual sources (e.g., near a particular musical instrument). This is a completely different problem because placing such microphones near individual sources with varying spectral characteristics results in signals whose frequency content will depend highly on the microphone positions. For spot microphones, we only considered their space dependence with respect to the orchestra and did not consider their dependence on hall acoustics. This allowed us to design timevarying filters (one for each spot microphone recording) that can enhance particular instrument types in the reference recording based on training datasets. For reverberant 
microphones, we only considered their dependence with respect to hall acoustics and did not consider the orchestra as a distributed source. This allowed us to design time-invariant filters (one for each reverberant microphone recording) that can add the reverberation effect in the reference recording, simulating the particular concert hall acoustic properties.

Spot microphones were treated separately by applying spectral conversion techniques for altering the short-term spectral properties of the reference audio signals. Some of the SC algorithms that have been used successfully for voice conversion can be adopted for the task of multichannel audio resynthesis quite favorably. In particular, three of the most common SC methods have been compared and our objective results, in accordance with our informal listening tests, have indicated that GMM-based spectral conversion can produce extremely successful results. Residual signal enhancement was also found to be essential for the special case of percussive sound resynthesis. Our current research has focused on audio quality improvement for the methods proposed here, by using alternative models for the short-term spectral properties of the audio signals. Other possible directions for future research include conducting formal listening tests, as well as extending the methods described here towards remastering existing monophonic and stereophonic recordings for multichannel rendering (the synthesis problem). Our experiments so far were conducted mostly with the chorus microphone case in mind. We also examined a special case of transient sounds, namely, percussive drum-like sounds, which are considered perceptually significant. Other types of transient sounds as well as various instrument types should be considered, possibly resulting in improved or novel algorithms.

For the reverberant microphone recordings, we have described a method for synthesizing the desired audio signals, based on spectral estimation techniques. The emphasis in this case is on the long-term spectral properties of the signals since the reverberation process is considered to be long in duration (e.g., two seconds for large concert halls). An IIR filtering solution was proposed for addressing the long reverberation-time problem, with associated long impulse responses for the filters to be designed. The issue of objectively estimating the performance of our methods arose and was treated by proposing the NMI as a measure of spectral distance that was found to be very suitable for comparing the long-term spectral properties of audio signals. The designed IIR filters are currently not suitable for realtime applications. We are investigating other possible alternatives for the filter design that will result in more practical solutions.

\section{ACKNOWLEDGMENT}

This research has been funded by the Integrated Media Systems Center, a National Science Foundation Engineering Research Center, Cooperative Agreement no. EEC-9529152.

\section{REFERENCES}

[1] A. Mouchtaris, Z. Zhu, and C. Kyriakakis, "High-quality multichannel audio over the Internet," in Proc. 33rd Asilomar Conference on Signals, Systems, and Computers, vol. 1, pp. 347351, Pacific Grove, Calif, USA, October 1999.

[2] S. Haykin, Adaptive Filter Theory, Prentice-Hall, Upper Saddle River, NJ, USA, 1996.

[3] D. W. Griffin and J. S. Lim, "Signal estimation from modified short-time Fourier transform," IEEE Trans. Acoustics, Speech, and Signal Processing, vol. 32, no. 2, pp. 236-243, 1984.

[4] M. Abe, S. Nakamura, K. Shikano, and H. Kuwabara, "Voice conversion through vector quantization," in Proc. IEEE Int. Conf. Acoustics, Speech, Signal Processing (ICASSP '88), pp. 655-658, New York, NY, USA, April 1988.

[5] Y. Stylianou, O. Cappe, and E. Moulines, "Continuous probabilistic transform for voice conversion," IEEE Trans. Speech, and Audio Processing, vol. 6, no. 2, pp. 131-142, 1998.

[6] A. Kain and M. W. Macon, "Spectral voice conversion for text-to-speech synthesis," in Proc. IEEE Int. Conf. Acoustics, Speech, Signal Processing (ICASSP '98), pp. 285-288, Seattle, Wash, USA, May 1998.

[7] G. Baudoin and Y. Stylianou, "On the transformation of the speech spectrum for voice conversion," in Proc. International Conf. on Spoken Language Processing (ICSLP '96), pp. 14051408, Philadephia, Pa, USA, October 1996.

[8] L. Rabiner and B.-H. Juang, Fundamentals of Speech Recognition, Prentice-Hall, Englewood Cliffs, NJ, USA, 1993.

[9] M. Slaney, "Semantic-audio retrieval," in Proc. IEEE Int. Conf. Acoustics, Speech, Signal Processing (ICASSP '02), pp. 4108-4111, Orlando, Fla, USA, May 2002.

[10] P. J. Moreno and R. Rifkin, "Using the Fisher kernel method for web audio classification," in Proc. IEEE Int. Conf. Acoustics, Speech, Signal Processing (ICASSP '00), pp. 2417-2420, Istanbul, Turkey, June 2000.

[11] A. Berenzweig, D. P. W. Ellis, and S. Lawrence, "Anchor space for classification and similarity measurement of music," in Proc. IEEE International Conference on Multimedia and Expo (ICME), Baltimore, Md, USA, July 2003.

[12] D. A. Reynolds and R. C. Rose, "Robust text-independent speaker identification using Gaussian mixture speaker models," IEEE Trans. Speech, and Audio Processing, vol. 3, no. 1, pp. 72-83, 1995.

[13] G. Strang and T. Nguyen, Wavelets and Filter Banks, WellesleyCambridge Press, Wellesley, Mass, USA, 1996.

[14] S. N. Levine, T. S. Verma, and J. O. Smith III, "Multiresolution sinusoidal modeling for wideband audio with modifications," in Proc. IEEE Int. Conf. Acoustics, Speech, Signal Processing (ICASSP '98), pp. 3585-3588, Seattle, Wash, USA, May 1998.

[15] R. J. McAulay and T. F. Quatieri, "Speech analysis/synthesis based on a sinusoidal representation," IEEE Trans. Acoustics, Speech, and Signal Processing, vol. 34, no. 4, pp. 744-754, 1986.

[16] X. Serra and J. O. Smith III, "Spectral modeling synthesis: a sound analysis/synthesis system based on a deterministic plus stochastic decomposition," Computer Music Journal, vol. 14, no. 4, pp. 12-24, 1990.

[17] O. Cappe and E. Moulines, "Regularization techniques for discrete cepstrum estimation," IEEE Signal Processing Letters, vol. 3, no. 4, pp. 100-102, 1996.

[18] J. Laroche and J.-L. Meillier, "Multichannel excitation/filter modeling of percussive sounds with application to the piano," IEEE Trans. Speech, and Audio Processing, vol. 2, no. 2, pp. 329-344, 1994.

[19] R. B. Sussman and M. Kahrs, "Analysis and resynthesis of musical instrument sounds using energy separation," in Proc. 
IEEE Int. Conf. Acoustics, Speech, Signal Processing (ICASSP '96), pp. 997-1000, Atlanta, Ga, USA, May 1996.

[20] M. W. Macon, A. McCree, W. M. Lai, and V. Viswanathan, "Efficient analysis/synthesis of percussion musical instrument sounds using an all-pole model," in Proc. IEEE Int. Conf. Acoustics, Speech, Signal Processing (ICASSP '98), pp. 35893592, Seattle, Wash, USA, May 1998.

[21] J. Laroche, "A new analysis/synthesis system of musical signals using Prony's method-Application to heavily damped percussive sounds," in Proc. IEEE Int. Conf. Acoustics, Speech, Signal Processing (ICASSP '89), pp. 2053-2056, Glasgow, UK, May 1989.

[22] H.-I. Choi and W. J. Williams, "Improved time-frequency representation of multicomponent signals using exponential kernels," IEEE Trans. Acoustics, Speech, and Signal Processing, vol. 37, no. 6, pp. 862-871, 1989.

[23] M. Mboup, M. Bonnet, and N. Bershad, "LMS coupled adaptive prediction and system identification: a statistical model and transient mean analysis," IEEE Trans. Signal Processing, vol. 42, no. 10, pp. 2607-2615, 1994.

[24] L. Ljung, System Identification: Theory for the User, PrenticeHall, Englewood Cliffs, NJ, USA, 1987.

[25] R. B. Blackman and J. W. Tukey, The Measurement of Power Spectra, Dover Publications, New York, NY, USA, 1958.

[26] J. W. Cooley and J. W. Tukey, "An algorithm for the machine calculation of complex Fourier series," Mathematics of Computation, vol. 19, no. 90, pp. 297-301, 1965.

[27] P. D. Welch, "The use of fast Fourier transform for the estimation of power spectra: a method based on time averaging over short, modified periodograms," IEEE Trans. Audio and Electroacoustics, vol. 15, no. 2, pp. 70-73, 1967.

[28] O. Shalvi and E. Weinstein, "System identification using nonstationary signals," IEEE Trans. Signal Processing, vol. 44, no. 8, pp. 2055-2063, 1996.

[29] J. T. G. Stockham, T. M. Cannon, and R. B. Ingebretsen, "Blind deconvolution through digital signal processing," Proceedings of the IEEE, vol. 63, no. 4, pp. 678-692, 1975.

[30] B. D. Radlovic and R. A. Kennedy, "Nonminimum-phase equalization and its subjective importance in room acoustics," IEEE Trans. Speech, and Audio Processing, vol. 8, pp. 728-737, November 2000.

[31] F. Itakura and S. Saito, "A statistical method for estimation of speech spectral density and formant frequencies," Electronics and Communications in Japan, vol. 53A, pp. 36-43, 1970.

[32] B. C. J. Moore, An Introduction to the Psychology of Hearing, Academic Press, New York, NY, USA, 1989.

[33] T. M. Cover and J. A. Thomas, Elements of Information Theory, Wiley, New York, NY, USA, 1991.

[34] D. B. Trizna, C. Bachmann, M. Sletten, N. Allan, J. Topokovand, and R. Harris, "Projection pursuit classification methods applied to multiband polarimetric SAR imagery," in Proc. IEEE International Geoscience and Remote Sensing Symposium (IGARSS '00), vol. 1, pp. 105-107, Honolulu, Hawaii, USA, July 2000.

[35] C. Shekhar and R. Chellappa, "Experimental evaluation of two criteria for pattern comparison and alignment," in Proc. 14th International Conference on Pattern Recognition (ICPR '98), vol. 1, pp. 146-153, Brisbane, Australia, August 1998.
Athanasios Mouchtaris received the Diploma degree in electrical engineering from the Aristotle University of Thessaloniki, Greece, in 1997 and the M.S. degree in electrical engineering from the University of Southern California (USC), in 1999. He is currently pursuing the Ph.D. degree at USC, working within the Immersive Audio Laboratory of the Integrated Media Systems Center. His research interests include signal processing for rendering immersive audio environments, contentbased audio enhancement for multichannel rendering, and audio synthesis for efficient transmission of multichannel recordings.

Shrikanth S. Narayanan received his M.S., Engineer, and Ph.D. degrees, all in electrical engineering from University of California at Los Angeles (UCLA) in 1990, 1992, and 1995, respectively. From 1995 to 2000, he was with AT\&T Labs Research, Florham Park, NJ (formerly AT\&T Bell Labs, Murray Hill)_first as a Senior Member and later as a Principal Member of its technical staff. He is currently an Assistant Professor in the Electrical Engineering Department, Signal and Image Processing Institute, University of Southern California (USC). He is also a Research Area Director of the Integrated Media Systems Center, an NSF ERC, and holds joint appointments in computer science and linguistics at USC. He is an Associate Editor of the IEEE Transactions of Speech and Audio Processing and serves on the Speech Communication technical committee of ASA. His research interests include signal processing and systems modeling with emphasis on speech, audio, and language processing. Shrikanth Narayanan is an author or coauthor of over 90 publications and holds 3 US Patents. He is a recipient of an NSF CAREER Award, a Center for Interdisciplinary Research Fellowship; he is a Member of Tau Beta $\mathrm{Pi}$ and Eta Kappa Nu and a Senior Member of IEEE.

Chris Kyriakakis received his B.S. degree from the California Institute of Technology in 1985 and his M.S. and Ph.D. degrees from the University of Southern California in 1987 and 1993, respectively. Since 1996, he has been in the faculty of Electrical Engineering Systems Department at USC and is currently an Associate Professor. He is the Director of the Immersive Audio Laboratory that is part of the Integrated Media

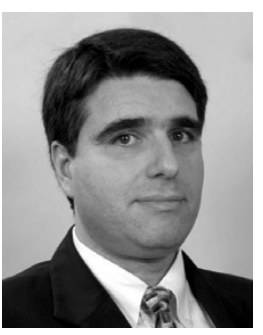
Systems Center, an NSF Engineering Research Center at the USC School of Engineering. His research interests include acquisition, synthesis, and rendering multichannel immersive audio, multiperson room equalization, microphone arrays, psychoacoustics, and error-free transmission of multichannel audio over high bandwidth networks. 